\title{
Correcting the Fabry-Perot artifacts in metamaterial retrieval procedures
}

\author{
Xing-Xiang Liu, ${ }^{1}$ David A. Powell, ${ }^{2}$ and Andrea Alù ${ }^{1, *}$ \\ ${ }^{1}$ Department of Electrical and Computer Engineering, The University of Texas at Austin, Austin, Texas 78712, USA \\ ${ }^{2}$ Nonlinear Physics Center, Research School of Physics and Engineering, The Australian National University, Canberra ACT 0200, Australia
}

(Received 13 June 2011; revised manuscript received 14 November 2011; published 1 December 2011)

\begin{abstract}
We discuss the well-known limitations in the retrieval of homogenized constitutive parameters based on reflection and transmission from metamaterial slabs arising near the Fabry-Perot resonances of the samples under analysis. The associated artifacts can significantly affect the retrieval results, causing Lorentzian-like nonphysical features. We discuss the nature of these artifacts and propose a general method to correct them based on a numerical regression procedure. These concepts are applied to a homogeneous slab and a periodic metamaterial array, showing that the proposed correction can provide an improved retrieval technique for metamaterial homogenization purposes.
\end{abstract}

DOI: 10.1103/PhysRevB.84.235106 PACS number(s): 42.70.Qs, 41.20.Jb, 78.20.-e, 42.25.Bs

\section{INTRODUCTION}

Metamaterials are structured materials composed of subwavelength inclusions that collectively exhibit exotic bulk properties $^{1-4}$ of interest in a variety of applications. The mechanisms that support these phenomena are based on the complex interaction of electric and magnetic effects supported by the engineered atoms in each unit cell. Due to their subwavelength granularity, it is highly desirable to define averaged field quantities and assign effective homogenized constitutive parameters, such as permittivity and permeability, to describe the metamaterial wave interaction, similar to what is usually done to describe natural materials. This process is generally possible when $d / \lambda \ll 1$, where $d$ and $\lambda$ are the typical unit cell size and wavelength in the metamaterial, respectively. This constraint ensures that the average fields vary sufficiently slowly across each unit cell to ensure that an averaged description is appropriate.

The proper definition of effective constitutive parameters plays a crucial role in the practical design and application of metamaterials and it has been at the center of relevant debates in recent literature. Conventional homogenization approaches, such as volumetric mixing rules,${ }^{5,6}$ are well suited for natural materials and mixtures but are often inaccurate for metamaterials with exotic electromagnetic properties. ${ }^{7}$ The reasons behind this inaccuracy often lie in various forms of nonlocality in metamaterials, as extensively discussed in several recent papers (see, e.g., Refs. 8-11 and references therein). Accurate homogenization procedures have been put forward to take these issues into account, which become particularly relevant near the inclusion resonances and/or when the array granularity is not negligible..$^{9-12}$

One of the simplest and most common approaches to homogenization, the Nicholson-Ross-Weir (NRW) retrieval method, ${ }^{13-15}$ is based on the assumption that we can describe a complex metamaterial within an a priori homogeneous model whose parameters may be retrieved from scattering measurements of a metamaterial sample. The retrieval method provides straightforward analysis and is particularly well suited to experimental setups, once the metamaterial sample is available and ready to be characterized and tested. Typically, retrieval procedures are applied to planar metamaterial slabs with finite thickness from which transmission and reflection coefficients are calculated and related to the metamaterial constitutive parameters.

As extensively discussed in Ref. 7, retrieval procedures have inherent limitations, usually associated with the initial assumption of simple homogenized models to describe complex metamaterial arrays, which cannot necessarily capture all the phenomena at the basis of their exotic properties. A more rigorous approach to homogenization often requires considering higher-order spatial dispersion effects that are not included in conventional retrieval procedures. As shown in Ref. 11, the retrieved parameters do not necessarily coincide with the local effective permittivity and permeability of a given metamaterial sample, as an inherent magnetoelectric coupling arises from weak spatial dispersion effects within the lattice, which is usually neglected in conventional retrieval procedures. However, in the usual case in which the excitation is outside the metamaterial sample, the retrieved parameters may be correctly used as an equivalent description of the metamaterial sample, which may properly capture its propagation and scattering properties. The retrieved parameters may then be used to model more complex metamaterial devices, as long as it is not expected that they have local properties. ${ }^{16,17}$

Despite their simplicity, the application of retrieval procedures has been extensively discussed in recent papers for two main reasons: (a) the difficulty in selecting the correct wave-number branch, particularly tricky when the wavelength in the metamaterial is significantly smaller than the total sample size, ${ }^{13,18,19}$ and (b) the occurrence of nonphysical artifacts arising near the Fabry-Perot resonances of the sample, i.e., when the thickness of the metamaterial slab is an integer multiple of half wavelength in the metamaterial. ${ }^{14,20,21}$ If the first issue may be resolved by inspecting the averaged field distribution inside the metamaterial or performing the retrieval for different slab thicknesses and selecting the correct solution branches that satisfy causality constraints, ${ }^{13,18-23}$ the nonphysical artifacts associated with slab resonances have been simply associated with numerical noise, amplified by the zeros of the reflection coefficient, ${ }^{19,20}$ without providing physical insights into this specific sensitivity or a general method to get rid of these artifacts. Since numerical or experimental noise is 
always present, the nonphysical spikes in retrieved parameters are unavoidable and can affect the retrieval procedure over moderately wide frequency regions, usually around the range where most interesting effects are observed in metamaterials, such as where the homogenized parameters are negative. These artifacts are usually associated with the slab FabryPerot resonances, and therefore the retrieved parameters are often sensitive to the total slab thickness, violating the very definition of homogenized parameters, which should not be affected by the size or shape of the metamaterial sample under analysis. Some variants of the NRW retrieval have been recently put forward to take care of these issues. In Ref. 20 the authors propose a reduced retrieval procedure that neglects magnetic effects in dielectric-only metamaterials, with evident limitations in the case of artificial magnetism or magnetic inclusions. Reference 21 suggests adding a lossy substrate to reduce the resonance effects at the root of these artifacts, but this technique may seriously affect the inherent resonances of the metamaterial and their correct retrieval. In addition, a change of substrate has been shown to affect the metamaterial parameters when spatial dispersion effects are strong. ${ }^{24}$

In this paper we investigate and characterize the physical nature of these retrieval artifacts and propose a general postprocessing method, based on least-squares regression, that corrects the retrieved parameters, restores a consistent homogenized equivalent description of the metamaterial, in the sense discussed above and in Ref. 25, for which the retrieved parameters do not depend on the thickness of the slab under analysis. This modification procedure is particularly relevant in the case of lossless or low-loss metamaterials, since in this case Fabry-Perot resonant artifacts most substantially affect the retrieval procedure. We apply our theoretical model to metamaterial samples composed of spherical magnetodielectric inclusions and compare our improved retrieval with the analytical equivalent parameters obtained in Ref. 11, in order to show that these concepts may be successfully applied to realistic metamaterial geometries in frequency windows where positive or negative parameters are expected.

\section{THEORETICAL FORMULATION}

Consider a homogeneous planar metamaterial slab described by its characteristic impedance $\eta$ and wave vector $k$ and excited by a plane wave at normal incidence. Its reflection $R$ and transmission $T$ coefficients (consistent with the scattering-matrix elements $S_{11}$ and $S_{21}$ ) are related to the secondary constitutive parameters of the slab as

$$
\eta^{2}=\eta_{0}^{2} \frac{(1+R)^{2}-T^{2}}{(1-R)^{2}-T^{2}}
$$

and

$$
\cos (k d)=\frac{1-R^{2}+T^{2}}{2 T},
$$

where $\eta_{0}$ is the characteristic impedance of the background material and $d$ is the slab thickness. These equations are at the basis of standard retrieval procedures, allowing us to extract the effective $\eta$ and $k$ from calculated or measured $R$ and $T$. If we assume that the metamaterial may be described by bulk permittivity and permeability, then these equivalent ${ }^{25}$ relative parameters are related to $\eta$ and $k$ by inverting the usual relations

$$
k=k_{0} \sqrt{\varepsilon \mu}, \quad \eta=\eta_{0} \sqrt{\mu / \varepsilon},
$$

under an $e^{-i \omega t}$ time convention, where $k_{0}$ is the background wave number.

The values of $k$ obtained from Eq. (2) yield multiple branches, giving rise to ambiguities in the retrieval process that may be resolved by inspecting the averaged field distribution in the metamaterial slab. ${ }^{14}$ In addition, to obtain physically meaningful parameters we are to choose proper signs for $\eta$ and $k$, based on the passivity conditions ${ }^{26} \operatorname{Re}[\eta]>0, \operatorname{Im}[k]>0$. Unfortunately, even after these issues are properly taken into account, further complications commonly arise, as described in the following.

During the retrieval process, the complex coefficients $R$ and $T$ are inherently associated with noise and small errors caused by experimental uncertainty and/or limited numerical precision. In addition, the array granularity may introduce uncertainties in the exact length of the sample ${ }^{19}$ and in the position of the reference planes at which the relative phase of the scattering parameters should be calculated, introducing additional, unavoidable errors. In numerical computations, these errors may be amplified by discretization, mesh limitations, frequency sampling, and numerical convergence. Finally, higher-order Bloch modes associated with the finite transverse granularity may introduce small numerical deviations in the phase of the scattering parameters, as compared to an ideal homogeneous slab of the same thickness. As discussed in Ref. 27, virtual interfaces may be considered to properly take into account these deviations, which effectively add small phase corrections to the retrieved scattering parameters. In most frequency regions all these common sources of error are manageable and the retrieval procedure is overall rather robust, but in the vicinity of the Fabry-Perot resonances of the metamaterial sample under analysis the retrieval procedure is inherently unstable because Eq. (1) tends to the ratio between two very small numbers, and any error may be drastically amplified. In this circumstance, nonphysical artifacts in the effective parameters are produced even for arbitrarily small but finite errors. ${ }^{19,20}$

In order to understand how small, uncontrollable errors in $R$ and $T$ may give rise to nonphysical features in the retrieved parameters, we have simulated with commercial software $^{28}$ the scattering coefficients from a homogeneous planar slab with known relative permittivity $\varepsilon_{r}=2, \mu_{r}=1$ and normalized thickness $k_{0} d$ in the range $0.5 \pi \sim 2.5 \pi$. We use finite-element frequency domain simulations with adaptive tetrahedral mesh size around $0.02 \lambda$ and a numerical accuracy of $10^{-12} .^{29}$ Of course, this simple scattering problem may also be exactly solved by applying the continuity conditions at the slab boundaries, and we compare in Figs. 1(a) and 1(b) the magnitude and phase of $R$ and $T$ obtained numerically and analytically.

As expected, the curves overlap with very good approximation, and the relative error in the numerical simulations, shown in Figs. 1(c) and 1(d), is limited to very small values over most frequencies. However, as $|R| \rightarrow 0$ around the Fabry-Perot resonances of the slab, the relative amplitude error has an 
(a)

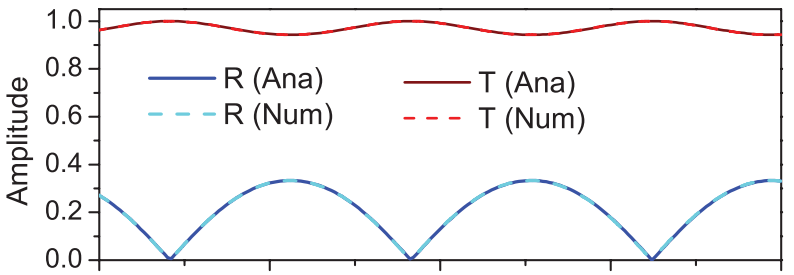

(b)
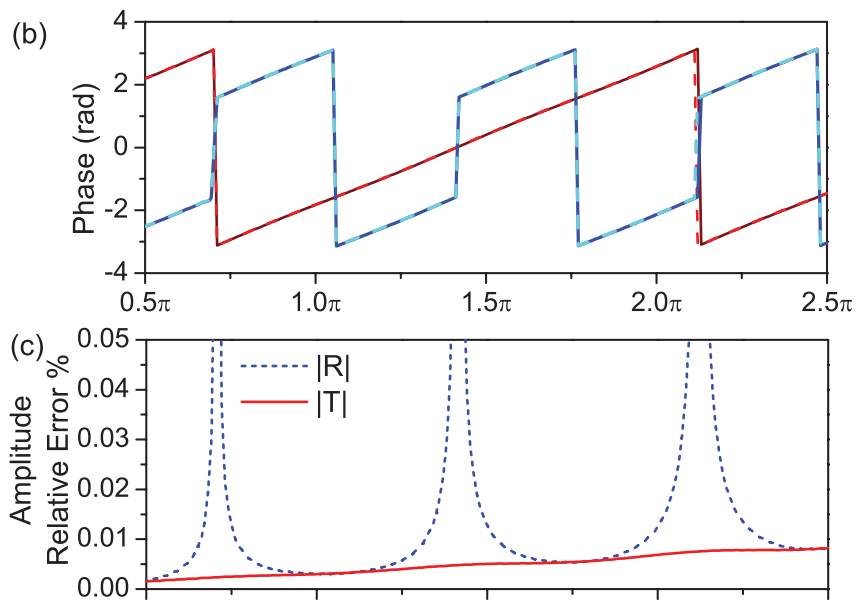

(d)

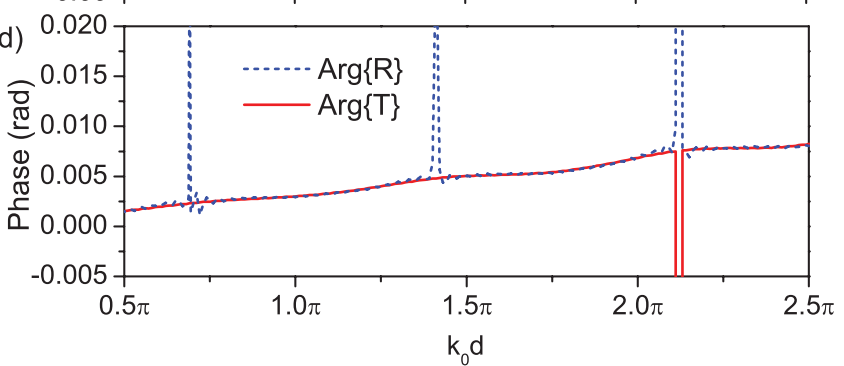

FIG. 1. (Color online) ( $a, b)$ Comparison of magnitude and phase of $R$ and $T$ derived analytically and with full-wave simulations for a planar slab with $\varepsilon_{r}=2, \mu_{r}=1$ (analytical and numerical curves are practically superimposed); (c) relative error magnitude; and (d) absolute phase error. The mesh size used in our finite-element numerical simulation is set at $0.02 \lambda$.

expected spike in the reflection coefficient, since very small quantities are being compared in this region. As we show in the following, these spikes may cause significant instabilities in the retrieved values of $\eta$. At the same frequencies, the phase error also shows associated spikes, as shown in Fig. 1(d). It is evident that a negligible shift in the calculated Fabry-Perot resonances of the sample, associated with the finite resolution of the mesh in our numerical simulations, causes a series of unavoidable singularities in Fig. 1(c).

We have applied a classic retrieval procedure to evaluate the effective parameters of the slab (as shown in Fig. 2), focused around one of the Fabry-Perot resonance frequencies of the slab for $k d=3 \pi\left(k_{0} d=2.121 \pi\right)$. It is seen that the effective index of refraction [Fig. 2(b), green line] correctly predicts a flat frequency dispersion all over the considered range, but the retrieved impedance [Fig. 2(a), green line] presents a Lorentzian-like artifact, precisely centered at this resonance frequency. This nonphysical feature in the retrieved impedance is then reflected in a similar Lorentzian spike in the retrieved permittivity and permeability, shown in Figs. 2(c) and 2(d). Notice that the divergence of the retrieved constitutive (a)
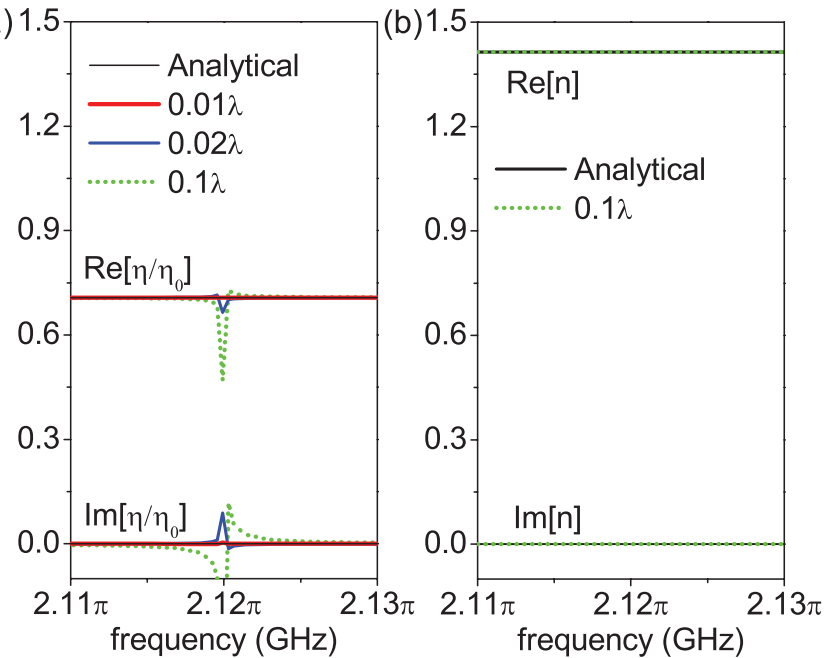

(c) 2
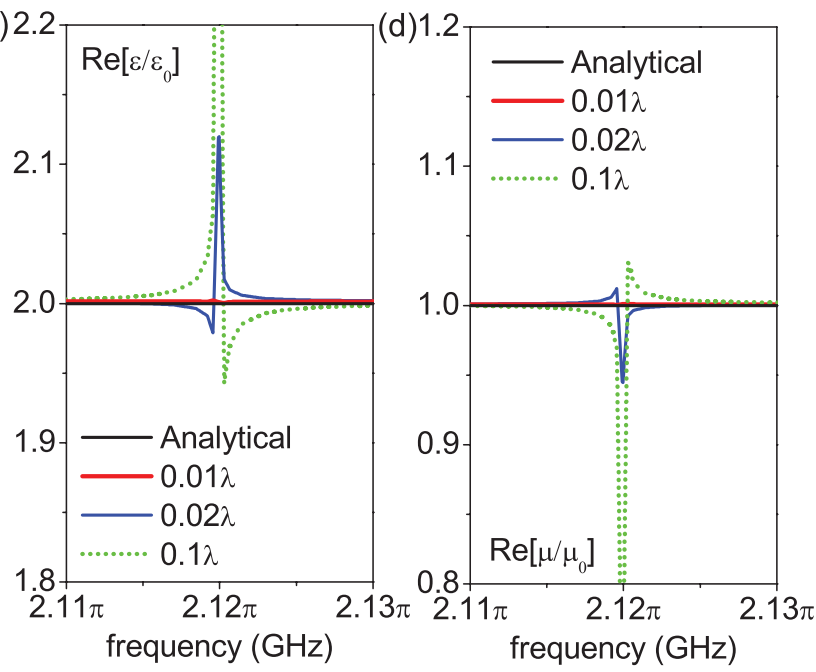

FIG. 2. (Color online) Retrieved impedance, index of refraction, permittivity, and permeability from the numerically simulated $R$ and $T$ with mesh sizes $0.1 \lambda, 0.02 \lambda$, and $0.01 \lambda$.

parameters from the correct value is not limited to the Fabry-Perot resonance frequency but extends over a finite range of frequencies in which errors of up to $10 \%$ may be obtained for both retrieved permittivity and permeability. In addition, the Lorentzian artifact in the retrieved permeability may have anticausal features, violating causality requirements associated with Kramers-Kronig relations, ${ }^{30}$ which require that in frequency windows with negligible losses, the conditions $\partial \operatorname{Re}(\varepsilon) / \partial \omega>0$ and $\partial \operatorname{Re}(\mu) / \partial \omega>0$ always apply and the material parameters grow with frequency. ${ }^{31}$ Similar artifacts are obtained also for the other Fabry-Perot resonances of the sample. In the same figure we have plotted similar curves for different mesh cell sizes to verify the effect of discretization on the Fabry-Perot artifacts. In particular, we have additionally considered mesh sizes of $0.01 \lambda$ and $0.1 \lambda$ to compute the retrieved parameters. It is evident that the artifacts are less and less relevant for more refined mesh sizes, but if one were to zoom near the resonance frequency, even for the most refined mesh some artifact would be visible. If the retrieval procedure fails to a non-negligible degree when applied to this ideal, homogeneous slab sample, the complexity of a realistic 
metamaterial sample may only worsen the accuracy of the retrieval procedure. It is also obvious from this discussion that the presence of substantial losses in the sample may smooth out these artifacts.

To gain some physical insights into the specific dispersion of these artifacts in the retrieved impedance, permittivity, and permeability associated with small errors in the scattering coefficients near a Fabry-Perot resonance, we Taylor expand the analytical expressions of $R$ and $T$ [Eqs. (1) and (2)] in the vicinity of an arbitrary resonance frequency at $k=k_{p}$ :

$$
\begin{aligned}
R_{\mathrm{num}}-R_{\mathrm{err}} & =R \approx R\left(k_{p}\right)+\frac{\partial R}{\partial k}\left(k-k_{p}\right) \\
& =-i \frac{\left(\eta^{2}-\eta_{0}^{2}\right)}{2 \eta \eta_{0}}\left(k-k_{p}\right) d,
\end{aligned}
$$

$$
\begin{aligned}
T_{\text {num }}-T_{\mathrm{err}} & =T \approx T\left(k_{p}\right)+\frac{\partial T}{\partial k}\left(k-k_{p}\right) \\
& = \pm 1-i \frac{\left(\eta^{2}+\eta_{0}^{2}\right)}{2 \eta \eta_{0}}\left(k-k_{p}\right) d .
\end{aligned}
$$

In the previous formulas $R\left(k_{p}\right)=0$ by definition, and the sign of $T\left(k_{p}\right)= \pm 1$ alternates at each Fabry-Perot resonance. We have defined complex coefficients $R_{\text {err }}$ and $T_{\text {err }}$ that take into account small deviations between the exact $R$ and $T$ and the numerically determined parameters $R_{\text {num }}$ and $T_{\text {num }}$. These errors may be associated with any of the previously mentioned sources of numerical or experimental uncertainties. We can safely expect these deviations to be slowly varying with frequency at around $k=k_{p}$. Using Eq. (1) we can calculate the retrieved impedance $\eta_{\text {num }}$ based on the values of $R_{\text {num }}$ and $T_{\text {num: }}$ :

$$
\begin{aligned}
\frac{\eta_{\mathrm{num}}^{2}}{\eta_{0}^{2}} & =\frac{\left(1+R_{\mathrm{num}}\right)^{2}-T_{\mathrm{num}}^{2}}{\left(1-R_{\mathrm{num}}\right)^{2}-T_{\mathrm{num}}^{2}}=1+\frac{4 R_{\mathrm{err}} \eta \eta_{0}-2 i d\left(k-k_{p}\right)\left(\eta^{2}-\eta_{0}^{2}\right)}{\left[\left(R_{\mathrm{err}}-T_{\mathrm{err}}\right) \eta+i d\left(k-k_{p}\right) \eta_{0}\right]\left[\left(R_{\mathrm{err}}+T_{\mathrm{err}}-2\right) \eta_{0}-i d\left(k-k_{p}\right) \eta\right]} \\
& \approx 1-\frac{2 R_{\mathrm{err}} \eta \eta_{0}-i d\left(k-k_{p}\right)\left(\eta^{2}-\eta_{0}^{2}\right)}{\eta_{0}\left[\left(R_{\mathrm{err}}-T_{\mathrm{err}}\right) \eta+i d\left(k-k_{p}\right) \eta_{0}\right]}
\end{aligned}
$$

where we have assumed $\left|k-k_{p}\right|,\left|R_{\mathrm{err}}\right|$ and $\left|T_{\mathrm{err}}\right|$ to be small. In the limit in which $R_{\text {err }}=T_{\text {err }}=0$, as expected $\eta_{\text {num }}=\eta$. However, even for very small errors, $\eta_{\text {num }}^{2}$ shows a complex nonphysical pole at $k d=k_{p} d+i \frac{\eta}{\eta_{0}}\left(R_{\mathrm{err}}-T_{\mathrm{err}}\right)$, which is responsible for a Lorentzian resonance in the retrieved impedance, centered around $k-k_{p}$. This artifact is unavoidable, unless the retrieval error is identically zero or negligible in a finite frequency range around $k_{p}$. We also notice that the weight of the Lorentzian artifact grows for larger values of $\eta / \eta_{0}$, making this issue particularly relevant near the metamaterial resonances or in regions in which extreme metamaterial parameters are obtained. Expanding in Taylor for small values of $R_{\mathrm{err}}$ and $T_{\mathrm{err}}$, we can also write

$$
\frac{\eta_{\mathrm{num}}^{2}}{\eta_{0}^{2}} \approx \frac{\eta^{2}}{\eta_{0}^{2}}+i \frac{\eta\left[\eta_{0}^{2}\left(T_{\mathrm{err}}+R_{\mathrm{err}}\right)-\eta^{2}\left(T_{\mathrm{err}}-R_{\mathrm{err}}\right)\right]}{\eta_{0}^{3}\left(k-k_{p}\right) d},
$$

which shows more explicitly the nature of the Lorentzian contribution in the retrieved characteristic impedance, associated with small errors in the scattering coefficients and located around $k=k_{p}$.

In the following section we modify the standard retrieval procedure and subtract from the retrieved $\eta_{\text {num }}$ the contribution associated with the second term in the right-hand side of Eq. (6) for each Fabry-Perot resonance of the slab. The modification procedure, described more extensively in the Appendix, is based on least-squares regression and can be generally applied to eliminate the Fabry-Perot artifacts, whatever their physical nature may be. In this way we are able to suppress the artificial Lorentzian pole and restore a smooth dispersion of the retrieved constitutive parameters that is independent of slab thickness and the position of the Fabry-Perot resonances of the sample under testing.

\section{IMPROVED RETRIEVAL PROCEDURE USING NUMERICAL REGRESSION}

We have shown in the previous section that a small error in the numerically extracted scattering coefficients around any Fabry-Perot resonance of an arbitrary metamaterial slab produces a Lorentzian pole in the retrieved characteristic impedance located at the frequency for which $k=k_{p}$. This explains and numerically quantifies the artifacts usually present in retrieved metamaterial parameters. It is evident from Eq. (6) that it may be possible to restore the proper metamaterial dispersion and remove these artifacts, provided that the values of $R_{\text {err }}$ and $T_{\text {err }}$ may be properly quantified. We use in the following a numerical regression technique based on the method of least squares to quantify the weight of $R_{\text {err }}$ and $T_{\text {err }}$ and remove these artifacts in a specific metamaterial geometry. Our numerical technique, based on the theory in the previous section, extracts the weights of each Lorentzian pole by estimating the complex value of $R_{\text {err }}$ and $T_{\text {err }}$. Then we can modify the standard retrieval procedure to remove the Lorentzian artifacts around each resonance, effectively subtracting from the original curve a sum of Lorentzian poles in the model of Eq. (6), with weights suitably determined at each Fabry-Perot resonance, as more extensively described in the Appendix.

We apply this technique to a planar slab composed of a cubic array of magnetodielectric spheres with permittivity $\varepsilon_{s}=13.8 \varepsilon_{0}$ and permeability $\mu_{s}=11.0 \mu_{0}$, as considered in recent papers to realize negative-index metamaterials at radio frequencies. ${ }^{11,12}$ The radius of each sphere is $a=0.45 d$, where $d$ is the array period and the total thickness of the slab is $8 d$. A sketch of one unit cell of this metamaterial is shown in the inset of Fig. 3(a). By applying standard retrieval procedures 

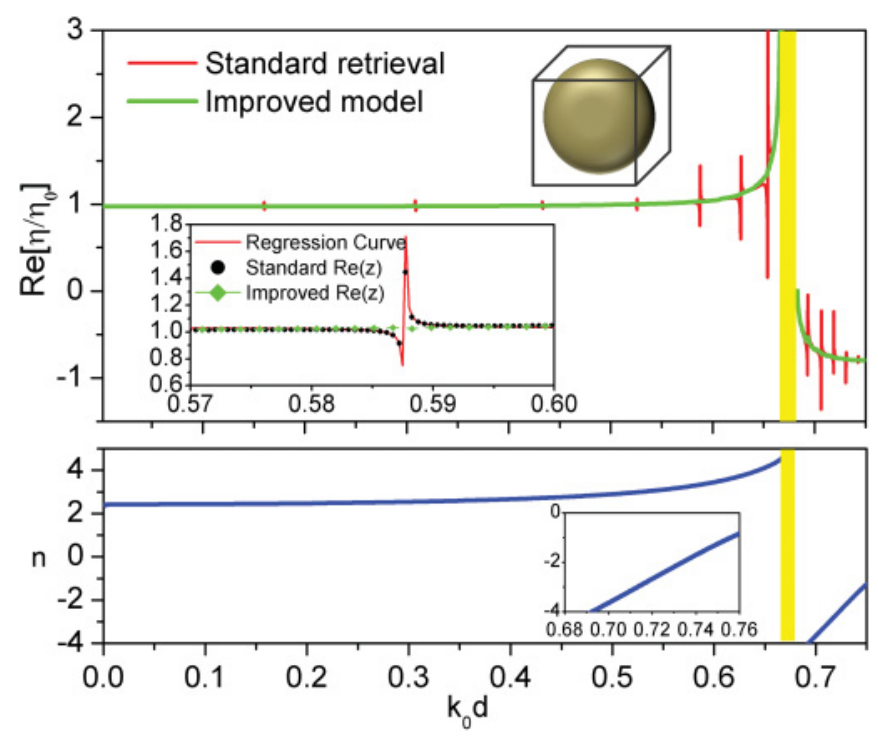

FIG. 3. (Color online) Dispersion of the retrieved effective impedance of a metamaterial slab composed of magnetodielectric spheres. The thin solid line shows the impedance retrieved with a standard procedure, whereas the bold line shows the modified retrieval obtained after removing the Lorentzian artifacts using the present theory. The inset shows the detail around one Fabry-Perot resonance, in which the original and modified retrieval curves are shown together with the fitted Lorentzian model.

to our numerical simulations, we have obtained the retrieved impedance, as shown in Fig. 3(a) (thin red line). The curve shows a series of Lorentzian spikes located at the Fabry-Perot resonances of the slab, consistent with Eqs. (5) and (6). The occurrence and relative weight of these spikes increase near the resonance of the inclusions, around $k_{0} d \simeq 0.67$, since the effective index of refraction increases in this frequency range and the Fabry-Perot resonances get closer together. Also, the weight of the Lorentzian spikes grows in this range of frequencies, since the ratio $\eta / \eta_{0}$ gets larger, consistent with the previous analysis. This is also related to an expected increased sensitivity to the discretization errors in this frequency range, since near the inclusion resonances the wavelength is largely reduced. Consistent with Eq. (6), the impedance also has a small imaginary part around each Lorentzian artifact (not shown here), a feature that is obviously not expected in a lossless metamaterial array.

The modified curve (thick green line), obtained after applying our regression method, shows a much smoother frequency dispersion, restoring the expected physical dispersion of the metamaterial impedance. In the figure the narrow shadowed region indicates the metamaterial stop band in which metamaterial homogenization and effective parameters lose their physical meaning.

In order to better clarify the nature of our proposed method, we show in the inset of Fig. 3(a) a zoomed detail of the previous curves around one of the Fabry-Perot resonances. In the inset, the black circles indicate the original retrieved values and the green squares show the modified curves. To remove the Lorentzian spike at around $k_{0} d=0.586$, we have used Eq. (6), shown as the solid red line in the inset, and we have subtracted the Lorentzian function from the retrieved curve.
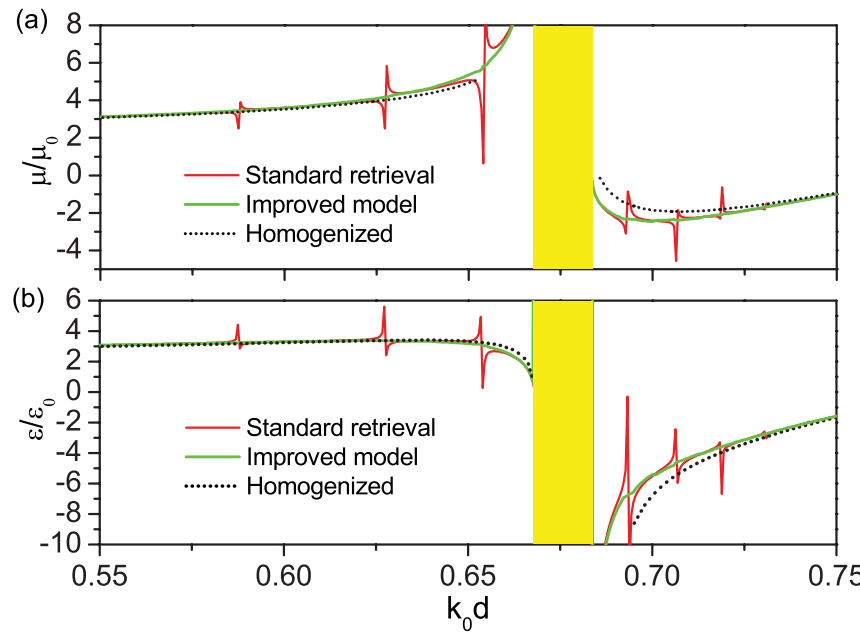

FIG. 4. (Color online) Effective permittivity and permeability corresponding to Fig. 3. The red thin lines show the retrieved parameters using standard retrieval techniques and the green bold lines show the modified curves. The yellow shadowed region indicates the metamaterial band gap, in which homogenized parameters lose their meaning, and the black dotted lines show the homogenized equivalent parameters predicted by the analytical theory presented in Ref. 11, based on dipolar approximations.

In this way we have been able to remove all the unphysical artifacts around the Fabry-Perot resonances of the slab and restore a smooth dispersion of retrieved impedance all over the range of frequencies of interest. Figure 3(b) shows the effective index of refraction retrieved in the same frequency range, which, as expected, does not show any spike artifact. We have used a technique similar to Ref. 14 to determine the correct choice of branch during the retrieval process. A negative-index region is found after the array stop band, as highlighted in the inset of Fig. 3(b).

In Fig. 4 we compare the parameters obtained with a standard retrieval procedure (thin red lines) and the curve obtained after the correction proposed here. In addition, we show in the same figure the analytical curves obtained for the equivalent constitutive parameters, as defined in the analytical homogenization theory introduced in Ref. 11 based on dipolar approximations. It is seen how the restored retrieved curve removes the artifacts at the Fabry-Perot resonances of the metamaterial sample, and the obtained parameters overlap with very good approximation with the analytical calculations based on the analytical homogenization results, despite that these curves are actually based on an approximate dipolar model. This result confirms the validity of the proposed retrieval procedure and ensures that the interaction among neighboring spheres is dominated by the dipolar fields. We stress that the analytical curves in Fig. 4 are based on the equivalent constitutive model, as defined in Ref. 11, which is valid to describe the scattering and propagation properties of source-free metamaterials and does not necessarily represent their first-principles effective homogenized model. ${ }^{25}$ The small nonzero imaginary part of the retrieved parameters (not reported here) is also removed by the proposed regression procedure, as expected in a lossless metamaterial array. 

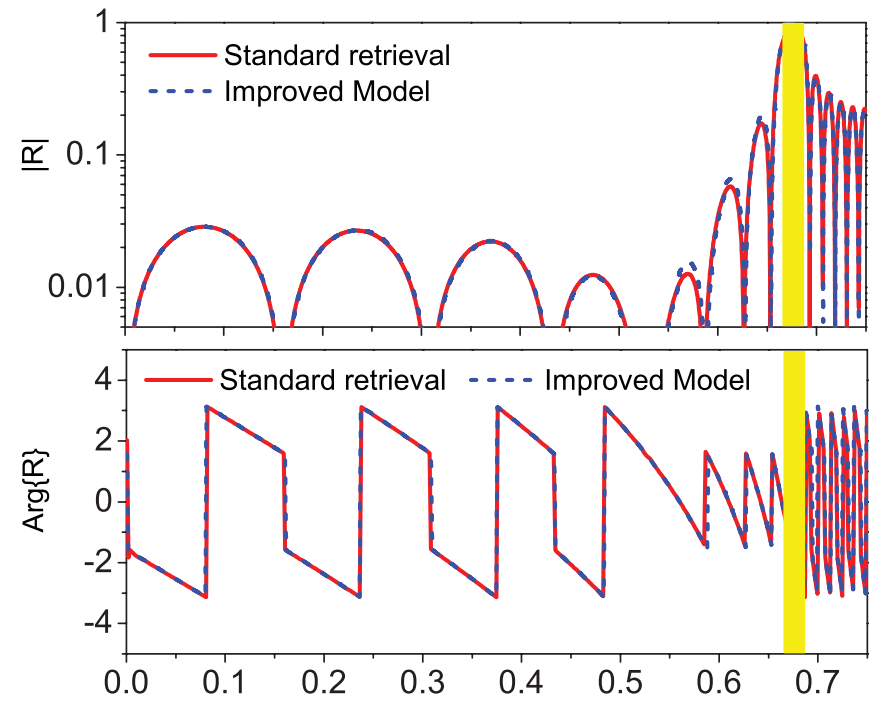

FIG. 5. (Color online) (a) Amplitude and (b) phase of reflection coefficients. The solid red lines present the original curves of standard retrieval obtained from full-wave simulations and the dashed blue lines are obtained inverting Eqs. (1) and (2) with the modified parameters corresponding to the improved model in Fig. 4.

It is remarkable to notice how the retrieved parameters, after proper correction, agree well over a wide range of frequencies with the homogenization theory for an infinite array, which intrinsically does not suffer from Fabry-Perot artifacts. The small differences are associated with the dipolar approximations in our analytical homogenization model and edge effects at the boundaries of the metamaterial slab. ${ }^{31}$ The proposed retrieval approach is very effective in all frequency windows, including the frequency range for which the effective parameters are simultaneously negative. We have verified that the restored curves (green lines) are not modified by the choice of a different slab thickness, despite the fact that the location and weight of the Fabry-Perot artifacts in the standard retrieval curves (red lines) are obviously affected.

Finally, Fig. 5 shows the reflection spectrum (in amplitude and phase) obtained from full-wave simulation and compared with the analytical curves obtained using Eqs. (1) and (2) and the restored permittivity and permeability curves in Fig. 4. The curves overlap over the entire frequency range considered, confirming the accuracy and consistency of this improved retrieval procedure, except for some negligible deviations near the stop-band region.

\section{CONCLUSION}

We have investigated here the nature of nonphysical artifacts in the dispersion of the effective constitutive parameters retrieved from the scattering properties of metamaterial slabs. These artifacts are the signature of Fabry-Perot resonances of the metamaterial samples and may be caused by small, unavoidable errors in numerical or experimental reflection and transmission data associated with discretization errors, unavoidable experimental noise, and/or the influence of higher-order Bloch modes below cutoff. They are relevant only near the slab resonances for low-loss metamaterials, in which the reflection coefficient tends to zero, and they affect only the retrieved characteristic impedance of the metamaterial, not its index of refraction. We have shown that in the limit of small errors, the artifacts have a specific Lorentzian dispersion, explaining the typically Lorentzian spikes commonly obtained in standard retrieval procedures. We have then proposed a numerical procedure based on a regression method to eliminate these spikes and restore retrieved parameters that do not depend on the slab thickness and have smoother frequency dispersion. Finally, we have successfully applied this technique to a metamaterial sample composed of magnetodielectric spheres, validating our approach. We reiterate that this technique does not aim at restoring the physical meaning of metamaterial retrieved parameters, which is dependent on the model a priori assumed in the retrieval method, ${ }^{7}$ but it aims at producing consistent and meaningful retrieved curves which are not affected by variations in the slab thickness or Fabry-Perot resonances of the specific sample under analysis. Similar concepts may be applied to more complex inclusions and experimentally retrieved data without particular complications.

\section{ACKNOWLEDGMENTS}

This work has been supported by the US AFOSR YIP Award No. FA9550-11-1-0009, the NSF CAREER Award No. ECCS-0953311, and by the ONR MURI Grant No. N00014-10-1-0942.

\section{APPENDIX}

In this Appendix we describe in more detail the numerical regression procedure proposed to extract the Lorentzian artifacts from the retrieved curves. This procedure may be generally applied to arbitrary metamaterial geometries, whatever the physical or numerical nature of the errors at the basis of the artifacts. Given a spectrum of transmission and reflection coefficients, we may obtain the effective refractive index and characteristic impedance by inverting Eqs. (1) and (2), as in a classical retrieval procedure. As described in the text, only the impedance curve will show Lorentzian artifacts, predicted at the frequencies for which $|R|$ is near zero. Therefore we need to apply the following numerical procedure to modify only the extracted values of $\eta$. Once a Fabry-Perot resonance frequency of the slab is identified, we apply the least-squares regression modification procedure in a frequency window around the resonance. The Lorentzian fitting function can be written as

$$
\varepsilon_{\infty}^{d} \pm \frac{\left(\omega_{p}^{d}\right)^{2}}{\left(\omega_{0}^{d}\right)^{2}-i \omega_{i} \gamma^{d}-\omega_{i}^{2}},
$$

where $\omega_{i}$ is a discrete sample frequency and the other dummy variables $\varepsilon_{\infty}^{d}, \omega_{p}^{d}$, and $\gamma^{d}$ with superscript $d$ model the predicted Lorentzian dispersion. The resonance frequency $\omega_{0}$ in the Lorentzian model corresponds to the Fabry-Perot resonance, consistent with Eq. (5). The \pm sign is chosen depending on whether a Lorentzian or anti-Lorentzian response is found. The free parameters in Eq. (A1) are used to fit the artifacts in the retrieved impedance curve. We extract a sufficient number of frequency samples around each Fabry-Perot resonance and 
aim at minimizing the expression:

$$
\min \sum_{i=-s}^{s}\left(\eta_{\text {ret }}\left(\omega_{i}\right)-\left[\varepsilon_{\infty}^{d} \pm \frac{\left(\omega_{p}^{d}\right)^{2}}{\left(\omega_{0}^{d}\right)^{2}-i \omega_{i} \gamma^{d}-\omega_{i}^{2}}\right]\right)^{2}
$$

where $\eta_{\text {ret }}\left(\omega_{i}\right)$ is the retrieved impedance obtained from Eq. (1) and $s$ is the number of frequency samples on each side of
Fabry-Perot artifact. The corrected impedance curve will then be obtained by subtracting from the classically retrieved curve a sum of optimally fitted Lorentzian functions on the model of Eq. (A1), centered around each Fabry-Perot resonance in the frequency range of interest with regressed dummy variables. Once the impedance curve is corrected, the retrieved permittivity and permeability curves will not contain any Fabry-Perot artifacts. *alu@mail.utexas.edu

${ }^{1}$ R. A. Shelby, D. R. Smith, and S. Schultz, Science 292, 77 (2001).

${ }^{2}$ J. Valentine, S. Zhang, T. Zentgraf, E. Ulin-Avila, D. A. Genov,

G. Bartal, and X. Zhang, Nature (London) 455, 376 (2008).

${ }^{3}$ N. Engheta and R. W. Ziolkowski (eds.), Electromagnetic Metamaterials: Physics and Engineering Explorations (John Wiley and Sons, New York, 2006).

${ }^{4}$ J. Li, L. Fok, X. Yin, G. Bartal, and X. Zhang, Nature Mater. 8, 931 (2009).

${ }^{5}$ A. Sihvola, Electromagnetic Mixing Formulas and Applications (IEEE Press, London, 1999).

${ }^{6} \mathrm{~F}$. Capolino (ed.), Theory and Phenomena of Metamaterials (CRC Press, Boca Raton, FL, 2009).

${ }^{7}$ A. Alù, Phys. Rev. B 83, 081102R (2011).

${ }^{8}$ C. R. Simovski and S. A. Tretyakov, Phys. Rev. B 75, 195111 (2007).

${ }^{9}$ D. R. Smith and J. B. Pendry, J. Opt. Soc. Am. B 23, 391 (2006).

${ }^{10}$ M. G. Silveirinha, Phys. Rev. B 75(10), 115104 (2007).

${ }^{11}$ A. Alù, A. D. Yaghjian, R. A. Shore, and M. G. Silveirinha, Phys. Rev. B 84, 054305 (2011).

${ }^{12}$ R. A. Shore and A. D. Yaghjian, Radio Sci. 42, RS6S21 (2007).

${ }^{13}$ D. R. Smith, S. Schultz, P. Markoš, and C. M. Soukoulis, Phys. Rev. B 65, 195104 (2002).

${ }^{14}$ X. Chen, T. M. Grzegorczyk, B.-I. Wu, J. Pacheco Jr., and J. A. Kong, Phys. Rev. E 70, 016608 (2004).

${ }^{15}$ D. R. Smith, D. C. Vier, Th. Koschny, and C. M. Soukoulis, Phys. Rev. E 71, 036617 (2005).

${ }^{16}$ X. X. Liu and A. Alù, J. Nanophotonics 5, 053509 (2011).

${ }^{17}$ X. X. Liu and A. Alù, Metamaterials 5, 56 (2011).
${ }^{18}$ V. V. Varadan and R. Ro, IEEE Trans. Microwave Theory Tech. 55(10), 2224 (2007).

${ }^{19}$ J. Woodley and M. Mojahedi, J. Opt. Soc. Am. B, 27, 1016 (2010).

${ }^{20}$ J. Qi, H. Kettunen, H. Wallén, and A. Sihvola, IEEE Antennas Wireless Propag. Lett. 9, 1057 (2010).

${ }^{21}$ U. C. Hasar, IEEE Antennas Wireless Propag. Lett. 20, 696 (2010).

${ }^{22}$ J. Zhou, T. Koschny, M. Kafesaki, and C. M. Soukoulis, Phys. Rev. B 80, 035109 (2009).

${ }^{23}$ S. Foteinopoulou, M. Kafesaki, E. N. Economou, and C. M. Soukoulis, Phys. Rev. B 84, 035128 (2011).

${ }^{24}$ D. A. Powell and Y. S. Kivshar, Appl. Phys. Lett. 97, 091106 (2010).

${ }^{25}$ The term equivalent is used in this context in the sense introduced in Ref. 11, in contrast with the effective constitutive parameters defined from first principles. The equivalent representation in terms of permittivity and permeability is useful to describe the propagation and scattering properties of the metamaterial in the absence of embedded excitation, ${ }^{7,11}$ but it does not necessarily imply that they possess inherently local properties.

${ }^{26}$ A. Alù and N. Engheta, IEEE Trans. Antennas Propag. 51, 2558 (2003).

${ }^{27}$ M. G. Silveirinha, A. Alù, and N. Engheta, Phys. Rev. E 75, 036603 (2007).

${ }^{28}$ CST Microwave Studio [www.cst.com].

${ }^{29}$ This number represents the relative residual error in the finiteelement simulation. Once the relative error gets below this number, the simulation has reached convergence.

${ }^{30}$ L. Landau and E. Lifschitz, Electrodynamics of Continuous Media (Butterworth-Heinemann, Moscow, Russia, 1984).

${ }^{31}$ C. R. Simovski, J. Opt. 13, 013001 (2011). 